\title{
Competencia para Estudiar y Aprender en Contextos Estresantes: Fundamentos de la Utilidad e-Afrontamiento del Estrés Académico
}

\section{Jesús de la Fuente ${ }^{1}$, Mireia López ${ }^{1}$, Lucía Zapata ${ }^{1}$, Jose Manuel Martínez-Vicente, Manuel Mariano Vera², Giulliana Solinas ${ }^{3}$, Salvatore Fadda ${ }^{3}$}

\begin{tabular}{c}
\hline${ }^{1}$ Universidad de Almería, España \\
${ }^{2}$ Universidad de Granada. España \\
${ }^{3}$ Universidad de Sassari, Italia \\
\hline
\end{tabular}

\section{España / Italia}




\section{Resumen}

La investigación sobre las emociones académicas en los procesos de enseñanzaaprendizaje universitarios ha cobrado fuerza en los últimos años. Tras la constatación de la importancia de las mismas, siguen siendo necesario modelos de evaluación e intervención. El objetivo de este informe es presentar el Modelo de Competencia para Estudiar, Aprender y Rendir bajo estrés, así como las variables específicas en las que se centra la evaluación e intervención, como fundamentos de la utilidad de e-Afrontamiento del Estrés Académico. También, se presentan las características esenciales de esta herramienta innovadora de autoayuda, útil para la auto-evaluación y la auto-mejora de los processos emocionales, asociados a las vivencias de estrés, en situaciones de aprendizaje universitario.

Palabras clave: estrés académico, modelo, auto-ayuda, afrontamiento, innovación, e-utilidad. 


\title{
Competency to study and learn in stressful contexts: fundamentals of the $e$-Coping with Academic Stress ${ }^{\mathrm{TM}}$ utility
}

\begin{abstract}
There has been growing research interest in achievement emotions in university teaching-learning processes in recent years. While their importance has been firmly established, there continues to be a need for assessment and intervention models. The objective of this report is to present the Competency Model for Studying, Learning and Performing under Stress, as well as the specific variables that underlie the assessment and intervention utility, e-Coping with Academic Stress. The basic characteristics of this innovative, self-help tool will be presented, as well as its utility for self-assessment and improvement in emotional processes that are associated with stressful experiences, in learning situations in higher education.
\end{abstract}

Keywords: academic stress, model, self-help, coping, innovation, e-utility 


\section{Introducción}

La investigación reciente sobre las emociones (positivas y negativas) durante los procesos de aprendizaje y rendimiento universitario ha puesto de manifiesto la relevancia de prestar atención a las mismas (Andersson et al, 2010; Bardi et al, 2011; Hamaideh, 2011). Los modelos cognitivos clásicos, de primera generación, procedentes de las metáforas del procesamiento de la información y del constructivismo, no han prestado suficiente atención a estas variables y al efecto de las mismas sobre los procesos cognitivos durante el aprendizaje. Los modelos motivacionales-afectivos de segunda generación (Pintrich, 2004; Zimmerman y Schunk, 2008, 2011) han insistido en la necesidad de prestar atención e intervenir sobre los procesos afectivos que operan durante el aprendizaje, por cuanto afectan y co-ocurren asociados -en unos casos de forma positiva y en otros de manera negativa e interferente- a los procesos cognitivos, que soportan el procesamiento de la información. Por ello, es esencial conocer las vivencias emocionales negativas propias del estrés, que concurren durante el aprendizaje universitario, en general, y de los estudiantes opositores, en particular (Regehr, Glancy y Pitts, 2013).

\section{La respuesta de estrés durante el aprendizaje y el rendimiento académico}

La respuesta de estrés en humanos ha sido ampliamente estudiada, en diferentes contextos, especialmente en el ámbito clínico y de la salud (Costarelli y Patsai, 2012; Hamdan-Mansour, Puskar, y Bandak, 2009; Gulewitsch et al., 2013; O'Donovan y Hughes, 2007; Pettit y De Barr, 2011). En el ámbito educativo, si bien se ha avanzado bastante en el conocimiento de los procesos cognitivos y metacognitivos, no se le ha prestado la atención suficiente para esclarecer con precisión los mecanismos que operan es esta respuesta, por su efecto pernicioso que puede operar en dichos procesos cognitivos y motivacionales-afectivos (de la Fuente, Zapata, Martínez-Vicente, Sander y Putwain, en prensa).

\section{El estrés académico}

Básicamente, el estrés es una respuesta adaptativa para producir un cambio en el organismo durante el proceso de ajuste al ambiente o contexto. En su origen, la respuesta de estrés se producía ante las situaciones de peligro para huir, protegerse o defenderse. En la actualidad, se produce -más probablemente- ante situaciones percibidas como potenciales de 
peligro o de amenaza psicológica. Por ejemplo, cuando un alumno cree que no le va a dar tiempo a estudiar todo el examen, cuando la tarea encomendada excede la percepción de sus posibilidades o cuando existen numerosas demandas de tareas a realizar, con la consiguiente sobrecarga de trabajo, estamos ante situaciones potenciales de estrés. Por tanto, es una respuesta general, aunque eminentemente fisiológica y asociada a otros procesos psicológicos: los pensamientos, los sentimientos y las acciones propios del estrés.

Existe evidencia de que la respuesta de estrés, en sus diferentes modalidades, interfiere en los procesos cognitivos y motivacionales durante el aprendizaje y el estudio (Serlachius, Hamer y Wardle, 2007). Por ello, tiene interés para el conocimiento de los procesos de aprendizaje, en general, y del estudio, en particular. Se ha constatado la interferencia con los procesos de memoria y de atención, así como de la recuperación de la información. La ansiedad asociada al estrés puede incrementar pensamientos de preocupación y emocionalidad negativa, lo que también puede afectar al estado motivacional-afectivo durante el aprendizaje, al interferir cambiando el curso de los pensamientos positivos con otros negativos y creencias irracionales, lo que puede autoinducir desmotivación (Largo-Wight, Peterson y Chen, 2005).

Por todo lo anterior, es muy importante trabajar en la prevención de las respuestas de estrés, instaurando las competencias necesarias de gestión del mismo en los alumnos, anticipándose a su aparición. Sin embargo, la mayoría de los Programas de Entrenamiento para Universitarios Estudiantes y Opositores al uso, obvia este aspecto, centrándose casi en exclusiva en las demandas de la oposición o del examen propiamente dicho.

\section{Indicadores del estrés académico}

La vivencia de estrés académico es multidimensional, tal y como se ha comentado. Este estado psicológico tiene diferentes componentes que funcionan como indicadores o correlatos del mismo: los pensamientos, las respuestas fisiológicas y el estado emocional son indicadores del mismo.

\section{1) Nivel cognitivo: los pensamientos asociados con el estrés}

En este nivel de respuesta el estudiante o el opositor pueden experimentar un incremento en la frecuencia, intensidad, duración, interferencia y situaciones en las que se producen pensamientos interferentes, en muchos casos de carácter automático y recurrente. 
Habitualmente son de carácter negativo, anticipatorias de fracaso, preocupación o con carácter auto-inductor de incapacidad para la tarea a realizar (Romero et al, 2014).

\section{2) Nivel fisiológico: la activación fisiológica y motora}

Este nivel de respuesta se refiere al incremento de diferentes respuestas fisiológicas en frecuencia, intensidad y duración, tanto durante el estudio, como respecto a las situaciones propias de examen (escrito u oral). Pueden estar referidas a múltiples sistema fisiológicos: sudoración, palpitaciones, insomnio, sequedad de boca, temblores, palpitaciones, etc. Los tipos de respuestas de tensión y ansiedad dependen de cada persona y de su historia de aprendizaje. Si un estudiante universitario u opositor tiene una historia de respuestas de este tipo, debería plantearse como un objetivo aprender a gestionar este tipo de respuestas, con suficiente antelación para que pueda reaprender a controlarlas. Es decir, no es buena idea, intentar aprender a relajarse o dejar de fumar dos días antes del examen, cuando uno ya nota mucha de la sintomatología comentada. Como cualquier reaprendizaje conductual se necesita tiempo y entrenamiento para poder modificar los comportamientos deseados.

\section{3) Nivel motivacional-afectivo: las emociones positivas vs negativas}

Este nivel de respuesta es muy importante, puesto que resume el estado motivacionalafectivo del estudiante o del opositor. En su vertiente positiva, éste tendría un estado de engagement (compromiso) con la tarea de preparación y estudio, es decir, está motivado, implicado en la tarea de estudio y el estrés negativo no le afectaría. Es decir, estaría en un nivel positivo de estrés, al traducir las demandas situaciones en un reto y con altas expectativas de éxito y energía para trabajar (Putwain, Sander y Larkin, 2013; Villavicencio y Bernardo, 2013). En su vertiente negativa, el estudiante u opositor tendrían un estado de burnout (agotamiento) o cansancio emocional que le impediría realizar sus tareas de manera óptima, con el consiguiente nivel de desmotivación y desafección por la tarea de estudio u oposición. Las personas que tienen este estado psicológico lo refieren como de agotamiento, falta de fuerza para afrontar las demandas situaciones o la sensación de estar "quemado" (Arsenio y Loria, 2014).

Obsérvese que las experiencias previas de éxito-fracaso académico o en la oposición pueden hacer más probable estar más cerca de un estado u otro. Por ejemplo, es razonable pensar que una persona que haya tenido varios fracasos en una asignatura u oposición, y que 
construya una idea de falta de posibilidades y de capacidad personal para ella, tenga un estado emocional negativo.

Esta evidencia y experiencia nos debe hacer reflexionar y tomar conciencia a los alumnos y opositores de que estudiar y rendir bajo presión requiere entrenamiento específico en conocimientos, habilidades, actitudes y hábitos de manejo del estrés, es decir, ser competente en la gestión del estrés (académico u opositor) durante el aprendizaje y el estudio, para conseguir el rendimiento deseado.

\section{Las situaciones de enseñanza-aprendizaje potencialmente inductoras de estrés}

Cualquier persona -y sobre todo, cualquier alumno- se ha enfrentado a alguna situación con potencial para generar estrés. Sin embargo, actualmente sabemos que las situaciones son estresantes de forma potencial, y depende, en gran medida, de la forma en que se afronten más que de la intensidad de los estímulos estresantes, por lo que que la forma de hacer frente del sujeto es más definitoria del impacto que los estímulos estresantes.

\section{Características del contexto de enseñanza-aprendizaje}

La investigación reciente ha establecido diferentes características de los contextos de aprendizaje que pueden ser potenciales inductoras de estrés:

1) La incertidumbre o falta de relación esfuerzo-resultado. Este es el caso de los estudiantes que necesitan estudiar y esforzarse durante mucho tiempo sin una garantía de éxito, a priori, en la consecución del los objetivos. Es decir, no existe una relación de contingencia lineal entre esfuerzo-resultado.

2) La percepción de que el sistema no es justo o fiable. Cuando la preparación ante la situación tampoco predice el resultado final de la misma. Esto ocurre cuando hay factores no controlables en la situación, por ejemplo, que se premie en el baremo determinados aspectos que algunos candidatos no pueden obtener (experiencia, años de servicio...), o que el profesor pueda tener diferentes criterios de corrección no explícitos. 
Existen determinadas características de la tarea de aprendizaje que puede llevar consigo un cierto nivel de estrés:

1) Las demandas de la situación. Si el contexto de aprendizaje demanda competencias a la persona que percibe que no tiene y no puede adquirir con facilidad (hablar en público, hablar en otro idioma, realizar un examen en un tiempo dado, solucionar un problema planteado, etc.). Estas tareas son complejas y no se adquieren mediante un mecanismo de ensayo y error, sino que requieren una combinación de construcción de conocimiento y la construcción de habilidades complejas.

2) La dificultad de la tarea. Cuando el contexto demanda un nivel de ejecución excelente en las tareas propuestas (sacar una calificación dada, rendir un mínimo en varias pruebas, etc.). En el nivel universitario, el estudio es, en sí mismo, una actividad estresante, por definición, habida cuenta del nivel y del volumen de la materia, así como de los sistemas de evaluación selectivos imperantes. Más aún ocurre en los contextos competitivos, en donde, además, se concursa y examina para la obtención de una plaza profesional.

3) La falta de una enseñanza reguladora. Este factor se refiere a la necesidad de que la persona tenga un guión de trabajo para poder operar durante el aprendizaje. Sin ese esquema o plan de trabajo, no tiene ayuda externa, para poder ir realizando logros y avances significativos, de manera gradual. Habitualmente las convocatorias de oposiciones o asignaturas, indican el producto final que hay que demostrar, pero no indican cómo llegar a ese nivel de competencia requerida (de la Fuente, García-Berbén y Zapata, 2013).

\section{Las características de la persona ante la situación}

Este nivel se refiere a las características personales que ayudan a gestionar el contexto y la tarea, es decir, al grado de competencia para estudiar y aprender en contextos de estrés En este esquema de análisis, esta variable es decisiva para opositar de manera efectiva. Es una competencia compleja que requiere tiempo de aprendizaje y entrenamiento específico. 


\section{La competencia para estudiar, aprender y rendir en contextos de estrés}

El concepto de competencia no es unidimensional, sino multidimensional (de la Fuente et al, 2004). Es decir, no se refiere a una sola habilidad o tipo de conocimiento. Más bien, se refiere al conjunto de conocimientos, habilidades, actitudes y hábitos que una persona ha construido, y que le permiten enfrentarse a una determinada situación de evaluación y salir airoso de la misma. Tal y como ha establecido el Modelo de Competencia para estudiar, aprender y rendir bajo estrés (de la Fuente, 2014a), ésta lleva consigo:

1) Saber (conocimiento factual). Este factor significa que la persona debe tener suficientes conocimientos factuales relativos a lo que significa la tarea de estudiar u opositar. Por ello, el estudiante debe poseer el conocimiento de:

- hechos relativos a los que significa la asignatura, carrera u oposción (requisitos, tipos de exámenes habituales, porcentajes de aprobados.

conceptos, referidos al conocimiento de la legislación, tipos de ejercicios, calificaciones, características de la asignatura o convocatoria y méritos exigidos. -principios, relativos a normas comportamentales y asunciones para estudiar $\mathrm{u}$ opositar.

2) Poder o saber hacer (conocimiento de habilidades y metahabilidades). Ello significa poseer:

-Las habilidades específicas de estudio (técnicas de estudio y estrategias de aprendizaje), así como las habilidades específicas para la oposición (escribir un examen, hablar en público, resolver problemas, etc.) y las habilidades de gestión del estrés (habilidades de relajación, ejercicio físico y de inhibición de la ansiedad evaluadora).

- También significa tener meta-habilidades de estudio (autorregulación del aprendizaje y estudio) y meta-habilidades de gestión del estrés (autorregulación personal, control del estrés, estrategias de afrontamiento del estrés, adecuados).

3) Querer o saber ser y saber estar (conocimiento de actitudes, valores y hábitos adecuados). Significa tener suficiente motivación e implicación en la asignatura, carrera u oposición para conseguir los logros y demandas planteadas. Este factor es decisivo puesto que afecta a los dos anteriores de una forma considerable. Ya se dice en el dicho popular español 
"más hace el que quiere que el que puede", lo que significa que la motivación es el "motor" del proceso de aprendizaje. Por ello significa, tener incorporados:

- Actitudes y valores para opositar, es decir, poseer un estilo motivacional de logro competitivo, con los demás (cuando se trata de un proceso selectivo, donde se compite con otros) o consigo mismo (en una asignatura, en donde el objetivo es la mejora o el logro personal). Asociado a ambas, es necesario tener creencias ajustadas y no irracionales, ya que afectan negativamente a la motivación y merman la motivación y el rendimiento.

- Los hábitos son esenciales. Tener buenos hábitos de trabajo y de estudio es nuclear para el logro de los objetivos y no perder la motivación. Los buenos hábitos garantizan un comportamiento disciplinado y eficaz en el aprovechamiento del tiempo, es decir, un ambiente estructurado de aprendizaje y estudio. Sin embargo, los hábitos sin los procedimientos de estrategias y meta-estrategias de estudio son insuficientes. Todos los aspectos mencionados se reflejan en la Tabla 1.

Tabla 1. Carácter multidimensional de la Competencia para estudiar, aprender y rendir bajo estrés (de la Fuente, 2014a; reproducido con permiso)

Hechos (conocimiento de las características de la asignatura o convocatoria; plazas, porcentajes de aprobados; requerimientos)

Saber Conceptos (sistema de trabajo, sistema de concurso-oposición, requerimientos; tipo de examen, puntuaciones, méritos previos)

Principios (creencias sobre las posibilidades u oposición y el sistema selectivo)

$+$

$\underline{\text { Poder }}$

Habilidades de aprendizaje y estudio (habilidades y técnicas de estudio)

Habilidades instrumentales (habilidades escritas y orales; inhibición de la ansiedad)

Meta-habilidades de estudio (estrategias de aprendizaje) y de gestión del estrés (estrategias de afrontamiento y autorregulación personal)

Actitudes y valores (confianza académica, motivación de logro)

\section{Querer}




\section{La evaluación e intervención para la mejora en el estrés académico}

La intervención psicoeducativa para ayudar a los estudiantes universitarios $\mathrm{u}$ opositores a manejar mejor su aprendizaje, estudio y estrés durante el mismo, se basa en el Modelo de Competencia para estudiar, aprender y rendir bajo estrés (de la Fuente, 2014a).

Variables de presagio: la experiencia previa de estrés

La experiencia previa sobre un determinado suceso estresante, hace probable determinados tipos de respuestas personales, según como haya sido esa experiencia previa.

1) En el caso de que el alumno no haya tenido experiencia previa en la universidad o en las oposiciones, el trabajo a desarrollar debe ser de carácter más preventivo. En este caso lo habitual es que la percepción de estrés sea más baja, ya que la experiencia aversiva de opositar es inexistente. Por ello, es deseable un entrenamiento en los diferentes subniveles competenciales para preparar al alumno para la experiencia que va a tener lugar.

2) En el caso existencia de varios ensayos de exposición a la experiencia de presentación a exámenes fallidos o a las oposiciones, lo habitual es que la percepción de desagradabilidad y de afección aumente significativamente respecto al grupo anterior

3) En el caso de que ya existan bastantes experiencias de presentarse a exámenes de una asignatura o a las oposiciones, generalmente la percepción de desagradabilidad y afección es la mayor de todas, por la historia de aprendizaje a la que el alumno ha estado expuesto. En algunos casos particulares, algunos estudiantes parecen quedar inoculados ante el estrés, por lo que esta experiencia puede producirles ya un menor nivel de afección. Por ello, este colectivo de alumnos "recurrentes" en el tiempo, debe ser tratado con especial atención.

Variables de proceso: la mejora de la competencia para estudiar y aprender bajo estrés

\section{Subcompetencias conceptuales}

1) Los hechos del exámen u oposición

La mejora de la información sobre los apartados ya comentados son específicos para cada asignatura o sistema de oposición. Las Convocatorias Oficiales y los materiales 
publicados por las diferentes editoriales ayudan a establecer una representación adecuada de todos los requerimientos de la misma. Un aspecto nuclear básico es que, en la mayoría de los casos, existe un doble proceso selectivo:

\section{El sistema de exámen u concurso-oposición}

Referido a las pruebas selectivas de la convocatoria. Pueden ser varios exámenes excluyentes o no, en unos casos escritos, en otros orales, en otros casos de desarrollo o pruebas profesionales, aplicadas según la Convocatoria. Es esencial conocer exactamente cado tipo de examen al que nos vamos a enfrentar, así como la puntuación de cada uno de los ejercicios.

\section{2) Los conceptos del exámen o concurso-oposición}

Los conceptos del examen u oposición están referidos al entramado complejo de conocimientos necesarios para la superar la prueba con garantía de éxito. Habitualmente están referidos al conocimiento académico-profesional pertinente para ejercer la profesión, a la que da acceso el proceso selectivo.

\section{3) Los principios del exámen u oposición: las creencias irracionales}

Las personas solemos elaborar ideas explicativas y proposiciones que nos permiten elaborar ideas propias de los acontecimientos de nuestra vida. En muchos casos, al ser elaboraciones personales, operan como principios explicativos de la realidad, por lo que llevan consigo posicionamientos personales o juicios de valor encubiertos. Si los principios son ajustados, nos benefician al contribuir al equilibrio psicológico, es decir a la emocionalidad positiva y a la motivación. Si los principios son desajustados, por ser irreales o irracionales, producen emocionalidad negativa y desmotivación. En situaciones de estrés, precisamente por la emocionalidad negativa de la situación es más probable que se elaboren este tipo de ideaciones negativas automáticas que operan como creencias personales o autoinstrucciones automáticas, a las que no prestamos atención, pero tienen un gran impacto en el estado emocional, y peor aún, en nuestra motivación. Existen muchos tipos de creencias irracionales que deben ser evaluadas y reajustadas, en el caso de que existan y se compruebe que están produciendo un efecto negativo en el estudiante opositor. Por ello, es conveniente su evaluación y trabajo posterior sobre las mismas. Si el estudiante tiene problemas para modificarlas o gestionarlas, debería acudir a un profesional Psicólogo Educativo. La intervención de mejora puede estar referida a: (1) bloqueo de este tipo de interferencias 
irracionales, mediante la técnica de parada de pensamiento; (2) reajuste y desmontaje de las mismas, mediante la reestructuración cognitiva. Se sugiere analizar con detenimiento, una a una, cuales están más instauradas y cuales están produciendo los efectos más nocivos en el mantenimiento de la motivación y del esfuerzo necesario para opositar.

\section{Subcompetencias procedimentales}

\section{Habilidades instrumentales del exámen o de la oposición}

Se entiende por habilidades instrumentales aquellas que son imprescindibles para la ejecución de los ejercicios del proceso selectivo. No es un aspecto menor, ya que los ejercicios se basan en las mismas. Habitualmente dependen de la historia de aprendizaje previa, pero, en cualquier caso, deben optimizarse para su puesta a punto, en los ejercicios profesionales.

\section{1) Habilidades de exposición escrita}

Un buen nivel de habilidad para el desarrollo de un tema o de un ejercicio práctico es esencial. Téngase en cuenta que las personas que evalúan los exámenes, no tienen conocimiento de lo que sabe el alumno, sino sólo de lo que ha escrito. Por ello, tras las evaluaciones efectuadas debes proponerte objetivos de mejora específicos y trabajar para mejorarlos. No de debe olvidar que las habilidades sólo se desarrollan si se practican.

\section{2) Habilidades de exposición oral}

Este tipo de habilidad es compleja y requiere un entrenamiento específico para cada oposición. No se expone igual un tema de un examen para Juez que un tema de Psicología que se realiza la defensa de una Programación Didáctica. La oposición es un buen momento para mejorarla. Tras la evaluación periódica, se encontrarán aspectos de fondo y forma a ir mejorando paulatinamente. Igual que en el caso anterior, se debe recordar que a hablar sólo se puede aprender hablando.

\section{Habilidades cognitivas}

\section{1) Estrategias de estudio y toma de apuntes}

Están referidos a los comportamientos estratégicos para hacer más eficiente el estudio y la toma de apuntes. En este apartado es muy importante que evalúes qué tácticas de estudio 
y toma de apuntes son correctas (uso de resúmenes, repetir en voz alta, anotaciones, búsqueda de ideas principales...) y qué aspectos te cuestan trabajo y podrías mejorar (organización del tiempo de estudio, selección de la información, ansiedad, ideas de fracaso....). Por tanto, la evaluación puede servir para plantearse objetivos de mejora en el estudio y la toma de apuntes.

\section{2) Estrategias cognitivas de aprendizaje}

Este aspecto se refiere a qué tipo de comportamientos específicos de procesamiento de la información utiliza el estudiante para construir el conocimiento mientras estudia, en tareas tales como la organización y selección de la información, la estructuración de la misma, la memorización o el resumen de la misma. Tras la evaluación podrá ser consciente de los aspectos que debe mejorar. Por ejemplo, si la memorización cuesta, se pueden aprender nuevas reglas nemotécnicas, o si el problema es la organización de la información, puede utilizar otra forma de organizarla mediante mapas conceptuales.

\section{3) Enfoques de aprendizaje}

Esta variable es una síntesis de las anteriores, porque dará información al estudiante de tu manera habitual en que lleva a cabo el estudio, si lo hace de manera profunda o se conforma con una forma superficial de hacerlo. Para la un exámen o sistema de oposición se podrá decidir en qué aspectos se debe utilizar un enfoque más profundo o más superficial, pero debe hacerse de manera consciente y programada por ti. De partida, un enfoque superficial, no ayuda a profundizar y a elaborar bien el conocimiento, por lo que es conveniente aproximarte a un enfoque más profundo que garantiza un conocimiento más elaborado y, sobre todo, una motivación por el estudio que ayuda mucho en esta situación de esfuerzo.

\section{Habilidades emocionales}

Las habilidades emocionales están referidas a aquellos procedimientos o tácticas para gestionar las emociones, convirtiendo las emociones negativas en positivas. Cuando las habilidades no existen o existe una historia de aprendizaje con percepción de falta de competencia en una determinada tarea, cualquier situación de evaluación puede convertirse en negativa, experimentando ansiedad y temor (Perera, Torabi y Kay, 2011). 


\section{1) Ansiedad de evaluación}

Es un tipo de emocionalidad negativa que afecta a la implicación y al rendimiento en la tarea, al producir pérdidas de recursos cognitivos y emocionales en quien la padece (Shamsuddin, et al., 2013; Zunhammer et al., 2013). Si la evaluación en la ansiedad ha puesto de manifiesto un nivel medio o alto, deberías aprender a controlarla y a sustituirla por emocionalidad positiva. Un cierto nivel de ansiedad ayuda a la ejecución y a mantener el estado de alerta; un exceso es pernicioso y puede ir asociado a otras alteraciones emocionales o de salud (depresión o agotamiento), especialmente, si se produce de forma mantenida, como es probablemente el caso de la preparación de oposiciones.

Meta-habilidades: habilidades meta-cognitivas, meta-afectivas y meta-comportamentales

Las meta-habilidades son habilidades de gestión o de control de las habilidades, ya sean éstas de tipo cognitivo, emocional o comportamental

\section{1) Estrategias de regulación cognitiva: el control del estudio}

Son habilidades de gestión del comportamiento cognitivo durante el estudio, con comportamientos adecuados antes (planificación y establecimiento de metas), durante (control y toma de decisiones) y después (reevaluación) del estudio. Este comportamiento, referido al comportamiento de autorregulación en el estudio ha sido ampliamente modelado (Benbenutty, Cleary y Kitsantas, 2014; Zimmerman y Labuhn, 2012).

\section{2) Estrategias de regulación emocional: las estrategias de afrontamiento}

Son habilidades de gestión del comportamiento emocional para gestionar y hacer frente al estrés que potencialmente produce el estudio y el aprendizaje en las situaciones de la universidad o en una oposición. Estas estrategias, según el modelo de Lazarus y Folkman (1968) pueden estar dirigidas a minimizar la emoción (centradas en la emoción) o el las causas que originan el problema (centradas en el problema).

Estas estrategias son especialmente relevantes porque permiten mantener el equilibrio emocional durante el período que dura todo en proceso de estudio y de oposición (Chou et al., 2011) o, en caso contrario, pueden ir asociadas a problemas de salud (Sulkowski, Dempsey y Dempsey, 2011). La evaluación proporciona una visión general de las estrategias de utilizas en mayor o menor grado, permitiéndo el ajuste estratégico de las mismas. Uno de los aspectos 
esenciales encontrados en la investigación reciente es que los alumnos universitarios utilizan en mayor medida estrategias dirigidas al problema, pero descuidan con cierta frecuencia, la gestión emocional, lo que a la larga puede pasar factura, especialmente, en períodos de estrés mantenido.

\section{3) Estrategias de regulación comportamental: la autorregulación personal}

Es la habilidad de gestión comportamental general (Pichardo et al, 2014) que permiten establecer planificar y establecer metas al comienzo de una tarea, establecer control y toma de decisiones durante la misma, y aprender de los errores, al finalizarla. Esta macrocompetencia personal es predictora de los comportamientos autorregulatorios en la vida cotidiana, lo que predice conductas de ajuste personal. Esta habilidad es especialmente relevante para el mantenimiento del esfuerzo personal y la disciplina en el trabajo y estudio durante períodos prolongados.

Subcompetencias actitudinales: actitudes, valores y hábitos

\section{Confianza académica}

Tras la evaluación de esta construcción psicológica se puede comprobar el nivel de confianza en uno mismo/a, respecto a la consecución de logros académicos y a la realización de comportamientos de estudio, de competencia verbal y de asistencia a las sesiones de clase. El nivel en la misma, que es consecuencia de la historia de aprendizaje, los alumnos que poseen un nivel bajo o medio, deberán trabajar para mejorar este aspecto (Sander et al., 2011; Sawatzky, et al., 2012).

\section{Motivación de logro}

La evaluación proporciona información respecto al grado de motivación de logro, es decir, del deseo y la necesidad de rendir bien y de conseguir logros académicos y profesionales. El nivel en el estilo de acción-emoción (de la Fuente, 2004, 2008; de la Fuente y Cardelle-Elawar, 2009, 2011; Lala, Bobirnac y Tipa, 2010; Salmerón, 2014), proporciona información del nivel motivacional-afectivo ante las situaciones de logro académico.

\section{Percepción o locus de control}

Esta variable te proporcionará información del grado en que tienes percepción de que los acontecimientos que te ocurren y los resultados que obtienes dependen de tus acciones y 
no de factores externos. Una mayor percepción de control lleva consigo mayor implicación y un mayor compromiso con la tarea de estudio y de esfuerzo.

\section{Resiliencia}

La resiliencia es la habilidad personal que permite superar y rehacerse ante las dificultades. Son muchas las situaciones de dificultad que un universitario estudiante $u$ opositor puede encontrarse a la largo de su proceso de estudio: desánimo, falta de motivación, resultados acordes con el esfuerzo o incidentes personales sobrevenidos (Artuch, 2014; Hartley, 2011).

\section{Hábitos de estudio}

Los hábitos son adquisiciones comportamentales, ensayadas de forma recurrente, que probabilizan en gran medida el futuro comportamiento de las personas, para bien o para mal. Los buenos hábitos de estudio están referidos a los comportamientos adecuados propios de la organización y la disciplina necesaria para llevar a cabo las horas de estudio necesarias con el fin de obtener un buen aprendizaje. Se diferencian de las estrategias de estudio porque aquellas están referidas a los comportamientos cognitivos que se producen en el estudio, mientras los hábitos son propios del comportamiento observable de estudio: el propio horario de estudio, el cumplimiento del mismo, o la elección del lugar adecuado.

\section{Variables de producto: la vivencia del estrés}

Como ya se ha comentado, las respuestas de estrés en humanos pueden afectar a diferentes niveles de conducta, pudiéndose encontrar diferentes correlatos o indicadores comportamentales de la vivencia de estrés (Burris, et al., 2009; Verger, Combes, KovessMasfety, et al., 2009; Tavolacci et al., 2013).

\section{Estrés cognitivo}

Referido a la frecuencia, intensidad y duración de pensamientos intrusivos o desajustados que aparecen a la par que las señales de tensión emocional. Estos pensamientos pueden ser causa o consecuencia de los otros niveles de estrés. La evaluación permite conocer el nivel de los pensamientos y si hay que realizar algún ajuste sobre los mismos. Los tratamientos psicológicos habituales utilizados para disminuirlos y ejercer control sobre ellos son las técnicas cognitivas para la reducción del estrés (McKay, Davis y Fanning, 1985), con una gama amplia de intervención según la característica de los pensamientos. 


\section{Estrés fisiológico y motor}

Referido a la frecuencia, intensidad y duración de respuestas de activación fisiológica. Este nivel de conducta es una señal directa del grado de estrés que está padeciendo. La evaluación permite detectar e intervenir en posibles señales o respuestas de estrés ante las situaciones de estudio o de la oposición. Existes numerosos tratamientos comportamentales para desactivar las respuestas de estrés fisiológico que son interferentes con el estudio o peligrosas para la salud, como la numerosa evidencia existente ha mostrado (Bodenlos, Noonan y Wells, 2013; Davis, McKey y Eshelman, 1985).

\section{Estrés emocional}

Referido a la frecuencia, intensidad y duración de las respuestas de agotamiento emocional o bournout (emocionalidad negativa) vs. compromiso o engagement con la tarea (emocionalidad positiva). La evaluación te permite conocer el nivel de emocionalidad negativa vs. positiva con la tarea universitaria de estudio u oposición, así como qué aspectos son ajustables. La investigación reciente ha destacado la importancia de la emocionalidad positiva (compromiso o engagement) durante el aprendizaje y estudio (Christenson, Reschly y Wylie, 2014; Conley, Travers y Bryant, 2013; Malinauskas, Malinauskiene y Dumciene, 2010), tras evidenciar de forma consistente el efecto pernicioso del agotamiento o bournout (Law, 2007). También se ha evidenciado la importancia de la utilización de técnicas o experiencias con mindfullnes (Caldwell, et al., 2010; de la Fuente, Franco y Mañas, 2010).

\section{La utilidad e-Afrontamiento del Estrés Académico}

La utilidad de e-Afrontamiento del Estrés Académico (de la Fuente, 2014b), http://www.estres.investigacion-psicopedagogica.com/espanol/seccion.php?idseccion=7 , está en consonancia con otros programas recientes de intervención (Regehr y Pitts, 2013) y de eintervención (Day, McGrath y Wojtowicz, 2013). Se puede utilizar de manera autónoma, pero se aconseja que se utilice con el asesoramiento pertinente de un Psicólogo Educativo. Las características específicas de la e-utilidad ya han sido expuestas en otro trabajo previo (de la Fuente et al, in review). 
La autoevaluación y mejora de la competencia para aprender, estudiar y rendir bajo estrés

Aunque existen una gran cantidad de inventarios de evaluación, en la utilidad de $e$ Afrontamiento del Estrés Académico (de la Fuente, 20014b) se han seleccionado aquellos que por sus características y diseño son sencillos para la autoaplicación, posibilitando la mejora de los comportamientos deseado. Para tal fin, se ha partido del modelo de Competencia para estudiar, aprender y rendir bajo estrés (de la Fuente, 2014a).

De manera coherente la e-utilidad permite establecer un nivel bajo-medio- alto del alumno evaluado, en cada variable del modelo dado. Dicho nivel ha sido calculado en una muestra previa de estudiantes universitarios u opositores -según el caso-, en los que también se han validado los inventarios previamente. No obstante, el nivel debe ser considerado sólo como un nivel referencial, y no algo absoluto, que ayude al estudiante o al opositor a tomar decisiones de mejora. Así, por ejemplo, un nivel bajo en una variable evaluada, indicaría que el estudiante debería trabajar bastante su grado de competencia en esa variable; un nivel medio, que estaría en el promedio de los estudiantes, pero debería mejorarla en cierta medida; un nivel alto, que tiene un buen nivel de competencia en la misma, pero puede mejorar determinados aspectos comportamentales. Un ejemplo del sistema de e-evaluación y eintervención para la mejora, respecto a la variable resiliencia, puede observarse a continuación. Ver Figuras 1, 2 y 3. 


\section{U.A.E. Utilidad de Afrontamiento del Estrés.}

Proyecto: Estrategias motivacionales-afectivas de autorregulación personal

y de afrontamiento del estrés, en el proceso de enseñanza aprendizaje universitario

Proyecto ref: EDU2011-24805 [2012-2014)

«Nivel: Alumnado

- Cuestionarios disponibles

- Ver mis resultados

- Guía de Autoayuda

- Ver o Editar mi ficha

- Cambiar mi contraseña

- Cerrar sesión
:: Alumno Opositor en pruebas ::.

..: Resultados detallados del Cuestionario: :t. .:. Escala de Resiliencia (OPO) ::. $\therefore(C D \cdot R I S C-O P O)=$

\begin{tabular}{|c|c|c|}
\hline Cuestionario Núm: 27 & \multicolumn{2}{|c|}{ G. Cumplimentado el: 21 de octubre de 2014} \\
\hline Titulo: & \multicolumn{2}{|l|}{ ESCALA DE RE SIUEENCIA (OPO) } \\
\hline Subritulo o Clave: & \multicolumn{2}{|l|}{ CD.RISC.OPO } \\
\hline Autories: & \multicolumn{2}{|c|}{$\begin{array}{l}\text { Connor-Davidsan (2003). Ingles \& Mateu-Pérez, Garcia-Renedo, } \\
\text { Caballer-Miedes y Gial-Beltran (2010) }\end{array}$} \\
\hline Problema que estudia: & \multicolumn{2}{|l|}{ Resiliencia } \\
\hline \multicolumn{3}{|c|}{ A) SUS RESULTADOS EN ESTE CUESTIONARIO: } \\
\hline Puntuación Global: & 3.85 [AIto] & Profuevan de Meion \\
\hline \multicolumn{3}{|c|}{ B) SUS RESULTADOS POR DIMENSIONES Y FACTORES: } \\
\hline \multicolumn{2}{|c|}{ 1. DIMENSIÔN: RESILIENCIA, 01: 3.80 [Aito] } & Hejos oe Dimocibn. \\
\hline \multicolumn{2}{|c|}{ 1. Factor Competencia Personal y Tenacidad, F1: 3.25 [Medio] } & Mkos abl Fuater \\
\hline \multicolumn{2}{|c|}{ 2. Factor Tolerancia al Estrés,F2: 3.06 [Medio] } & Mevora ov Fodst \\
\hline \multicolumn{2}{|c|}{ 3. Factor Tolerancia al Cambio, F3: 4.20 [Alto] } & 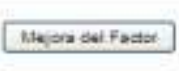 \\
\hline \multicolumn{2}{|c|}{ 4. Factor Percepción de Control, F4: 3.67 [Medio] } & 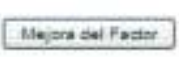 \\
\hline \multicolumn{2}{|c|}{ 5. Factor Influencia Espiritual, F5:5.00 [Aito] } & Miseris seat fostor \\
\hline \multicolumn{2}{|c|}{ 2. DIMENSION: METAEVALUACIÓN, D2: 5.00 [Alto] } & Mejera de Dimanisen \\
\hline \multicolumn{2}{|c|}{ 6. Factor Evaluaciön Inicial, F6 : 5.00 [Alto] } & Mesare bei Fostre \\
\hline \multicolumn{2}{|c|}{ 7. Factor Evaluación Procesual, F] : $0.00[$ Cero] } & 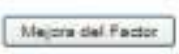 \\
\hline & Venter & \\
\hline
\end{tabular}

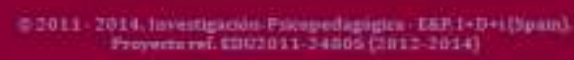

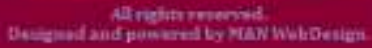

Figura 1. Ejemplo de resultado de un universitario opositor en el cuestionario de Resiliencia 


\section{U.A.E. Utilidad de Afrontamiento del Estrés.}

Proyecto: Eatrategias motivacionales afectivas de autorregulación personal

«ivel: Alumnado $\%$

- Cuestionarios disponibles

- Ver mis resultiadios

- Guía de Autoayuda

- Ver a fiditar mi fielua

- Cambiar mi contrasena

- Cerrar sesión
:Alumno Opositor en pruebas ::

in Propuestias de Mejora en función de los Resultados: :

Escala de Resillenela (OPO) (CD-RISC-OPO)

\begin{tabular}{|c|c|}
\hline Cunutionario Müm: 27 & 口. Propuenata de Mejara: 225 \\
\hline Tatulat & ESCAL A DE RE SILIERCIA (OPO) \\
\hline Subtitule o Claves: & CD-AISC-ORO \\
\hline Autaeriex: & 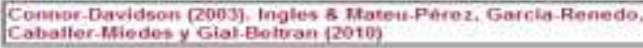 \\
\hline Pratinma que enturden: & Resilinneia \\
\hline
\end{tabular}

PROPUESTAS DE MEJORA PARA

Factor:

TOLERANCIA AL ESTRES,F2:[Medio]

La puntuaciòn obtenida te debe hacer reflexionar sobre esta caracteristica parsonel ante lit:

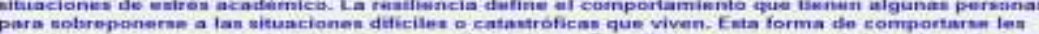
permile sobreponerse a taies situaciones y salir ndelante. Por ello, os una carscierimica adaptativa que

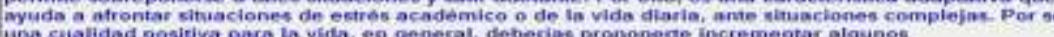
una zualldad positiva paro la vida, en act

Básicamente. la reailiencia se defino como un coniunto de comportamientos. referidos a diferentes aspectos: (1) percepeión de competencia y de tunacidad, (2) tolerancia al estres, (3) tolerancia al cambie.

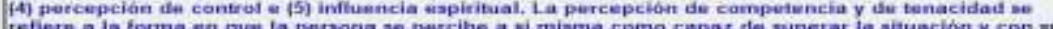

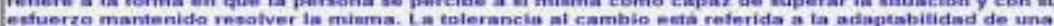
perenna antr situaciones cambiantes, a veces no desurdan. Le percepcion de control es propia de las personas que tienen lo creencia de que pueden realizar acciones para ejercer caubios en su entorno. Finalmente, lo intluencia espiritual se reflore a lo creencia de ayuda por parte de un ser waperior, o a ios creencias en motivos superiores para actuar.

Cortamiento, sine más bien un complejo de diferentes tipos de comporimimientos. Esta manera de reaccioenar no se aprende de repente, sino a travis de una expuriencia de apresdizaje comsolidadia en al tiempo y favorecida por caracteristicas personales an is Ani, podras comenzar a tratiajar para mejorar loe mpectos enpecificon que tengase man tajok. 1) sil aparecos imedio en la percepclon de competencia y tenacidad, deberias planiearto aumentarlis. La alumino, de les experiencias previas de exito fracaso y de las calificacioenes obtenidas. Es decir, si has

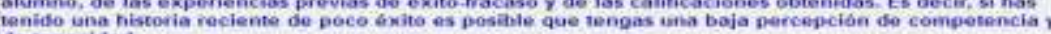
de tenacided.

Cunseguit murnemarta mediante diferenter actividade:

- Otsaerva y haz un lintado de tus competancias relacionadas con el matudio. Ondena de mayor a munot - Planea la mejora de tan que tienes mas hajar. Date sutsinatrusciones de mejara. - Enumera las careas on las que quieres ser man tenez.

- Si cempruebas que no puedes modificartos, pide ayude profosionat.

2) Si aparecen medio en tolurancia al estrés detiea mejorar este aspecto de tu forma de afrontar fas

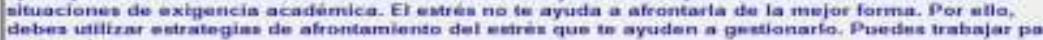

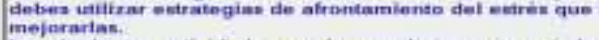

Las siguientes actividades pueden ayuderte a aumentarla

- Debes asumir que el estrés es consuntancial a lo vide acodémica por to exigencia que Heva consigo.

Dobes convencerte de que puedet ejercer control sobre el mismo,

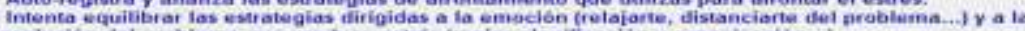
aciàn y arganización...

- Utiliza ia acoptacián si el proble

St no lngras reallear of aspecto anterior solota, bueca ayuda protesional

3) St apareces medio en la tolerancis al cambio debes mejorar eoto mpocto de tu forma de percithir tes acilided pora adeptarse a los imprevistos y cambios de todas las organizacioees. Por ello, detes vailizer

estroteglos pasa mejorarla,
Los siguiatentes actividades pueden ayudartu a aumentarla.

Deban convericarte que la capacidad de acuphar los cambios de la vida ayuda a duaenvolverne por ella. Auta registra y analiza ian sitizaciacion de cambios quo to prowacian inqui

- Plensa cada dia aspectos de ta vida que puedes cambior y baz pequenos ensaycis de cambio, fllo te

Si no logras realizar el aspecto anterior solovis, busca ayude profesional

4) Si apareces medio on la percepcíon de control debes mejorar este esvecto de tu forma de percibir les

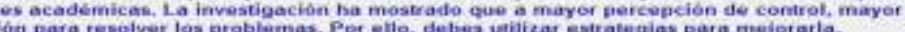

wa siguientena actividades pueden ayudarto a aumatitaria:

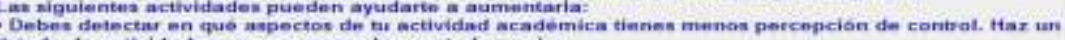
Stado de aclividades qun creas puedes controlar mejpe.

aut crees que puedes o no ejercer control notye

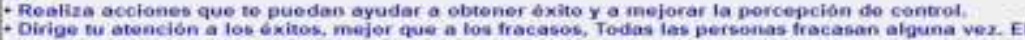

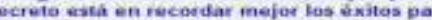

- Si no lopras realizar ef appecto antartior sotola, bunca ayuda protuaional.

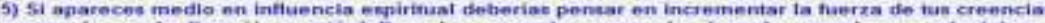
personsies, on la dirección que to definas, Las creencias personales, basadas en valores o principion

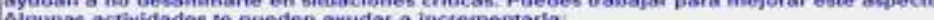

- Haz un listade de creencias o valores personales relovantes pare ti.

- Elatora una lded detenida do como to dyusiarian en vituaciones limites.

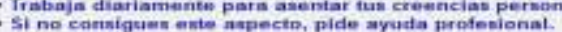

Figura 2. Ejemplo de propuestas de mejora en Resiliencia para alumnado opositor 


\section{U.A.E. Utilidad de Afrontamiento del Estrés.}

Proyecto: Estrategias motivactonales-afectivas de autorregulación personal

y de afrontamiento del estrés, en el proceso de ensenanza-aprendizaje universitarto

Proyecta ref EDU201124805 (2012.2014)

\section{«Nivel: Profesorado»}

- Datos por Alumnos

- Datos por Cuestionarios

- Guía de Autoayuda

- Ver o Editar mi perfil

- Cambiar mi contraseña

- Cerrar sesión
.: Sesión abierta por D./Dña. Jesús De la Fuente Árias ::.

$\therefore$ Resultados del Cuestionario:

"CUESTIONARIO DE ESTRATEGIAS DE AFRONTAMIENTO (UNI)" (Cumplimentado 321 veces)

\begin{tabular}{|c|c|c|}
\hline Cuestionario Núm: ? & \multicolumn{2}{|c|}{ G Cumplimentado por ùltima vez al: 28 de noviumbere de 2014} \\
\hline Titule: & \multicolumn{2}{|c|}{ CUE STIONAARIO DE ESTRATEGIAS OE AFRONTAMIENTO (UMI) } \\
\hline Subtitula o Clave: & \multicolumn{2}{|l|}{ EEC-IIII } \\
\hline Autories: & \multicolumn{2}{|l|}{ Chorot y Sandin (1963) } \\
\hline Problema que estudila: & \multicolumn{2}{|l|}{ Estrategias do Afrontamiento del Estres } \\
\hline \multicolumn{3}{|c|}{ A) SUS RESULTADOS EN ESTE CUESTIONARIO: } \\
\hline Puntuación Global: & 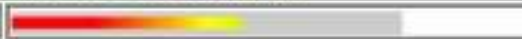 & 2.60 [Atedio] \\
\hline \multicolumn{3}{|c|}{ B) SUS RESULTADOS POR DIMENSIONES Y FACTORES: } \\
\hline \multicolumn{2}{|c|}{ 1. DEMENSIONL AFRONTAMIENTO CENTRADO EN LA EMOCION, D1: } & 2.24 [Bajo] \\
\hline 3 -Factor & Distracción fantistica, F1: & 2.29 [Medio] \\
\hline 4-Factor & Apoyo religioso , $\mathrm{F} 4:$ & 1.94 [Bajo] \\
\hline 6. Factor & Ayuda pare la acciòn, F6: & 2.35 [Media] \\
\hline 7 - Factor & Reducción de la ansiedad y evitación, FT: & 2.51 [Medio] \\
\hline 8. Factor & Preparación para lo peor, F8: & 2.70 [Dedio] \\
\hline 9-Factor & Descarga emocional y aislaminnto, F9: & 1.88 [Bajo] \\
\hline 11 - Factor & Aceptación resignada, F11: & $2.04[$ [Bajo] \\
\hline \multicolumn{2}{|c|}{ 2. DEMENSIONE AFRONTAMIENTO CENTRADO EN EL PROBLEMA, D2: } & 2.93 [Medio] \\
\hline 1 - Factot & Acciones dirigidas a las causas, F3: & 2.53 [Medio] \\
\hline 2. Factor & Bùsqueda de ayuda, F2: & 2.94 [Medio] \\
\hline 5-Factor & Autoinstrucciones, F5: & 3.09 [Medio] \\
\hline 10 - Factor & Reevaluación positiva y firmeza, F10: & 3.12 [Medio] \\
\hline 12 - Factor & Comunicación de sentimientos y apoyo social, F12: & 3.01 [Bedio] \\
\hline 13- Factor & Búsqueda de refuerzos alternativos, F13: & 2.86 [Medio] \\
\hline \multicolumn{2}{|c|}{ 3. OLMENSION: METAEVALUACIÓN, D3: } & 3.59 [Alto] \\
\hline 14 - Factor & Evaluación Inicial, F14: & 3.57 [Alto] \\
\hline 15 - Factor & Evaluación Proceseal, F15: & 3.62 [Alto] \\
\hline & velue & \\
\hline
\end{tabular}




\section{Conclusión}

Las vivencias emocionales durante el aprendizaje y el estudio, especialmente en las situaciones bajo presión y estrés potencial, son de tanta relevancia como los procesos cognitivos que se producen durante el mismo. Tradicionalmente, la evaluación psicoeducativa sobre el estudio y el aprendizaje, se ha centrado en el análisis de las habilidades cognitivas y estrategias metacognitivas para ayudar a los estudiantes. Esta idea es coherente en contextos de aprendizaje con poco nivel de estrés. Sin embargo, en los contextos de enseñanza-aprendizaje universitarios, en los que se dan las condiciones para que las respuestas de estrés se produzcan (alto nivel de exigencia de las tareas, alto nivel de rendimiento exigido, esfuerzo continuado e incertidumbre del logro de los objetivos) es necesario analizar no sólo los comportamientos cognitivos, sino también los emocionales.

En el caso específico de alumnado universitario, estudiante u opositor, es conveniente trabajar sobre un modelo de competencia para aprender y estudiar en situaciones de estrés (de la Fuente, 1994, 2014) que integre ambos niveles de subcompetencias conceptuales, procedimentales y actitudinales. Para ello, la Guía de uso (de la Fuente, 2014a) y la innovación tecnológica denominada e-Afrontamiento del Estrés Académico (de la Fuente, 2014b) pretenden contribuir a tal fin. En la actualidad, esta línea de investigación e intervención con universitarios tiene gran relevancia, como lo muestran otras utilidades desarrolladas por otros equipos de investigación de nuestro entorno (Buckingham y Dearin, 2012; Small y Deakin, 2008; Williamson et al., 2011).

\section{Agradecimientos}

Este informe de investigación, el Manual de uso y la utilidad e-Afrontamiento del Estrés Académico han sido elaborados gracias a la ayuda del Proyecto I+D ref. EDU201124805 (2012-2014). Ministerio de Ciencia e Innovación (España) y Fondo Social Europeo. Esta utilidad puede adquirirse para su uso, a través de la Empresa de Base Tecnológica de la Universidad de Almería Education \& Psychology $I+D+i$ (www.education-psychology.com) o contactando vía e-mail: lucia.epsychology@gmail.com; juente@ual.es 


\section{Referencias}

Artuch, R. (2014). Resiliencia y autorregulación en jóvenes navarros con riesgo de exclusion social. Tesis Doctoral sin publicar. Pamplona: Universidad de Navarra.

Andersson C, Johnsson KO, Berglund M, et al. (2010). Stress and hazardous alcohol use: associations with early dropout from university. Scand J Public Health, 37(7), 7137199. http://dx.doi.org/10.1177/1403494809344359

Arsenio, W.F. y Loria, S. (2014). Coping with negative emotions: connections with adolescents' academic performance and stress. J Genet Psychol, 175(1-2), 76-90. http://dx.doi.org/10.1080/00221325.2013.806293

Bardi, M., Koone, T., Mewaldt., S. et al. (2011). Behavioral and physiological correlates of stress related to examination performance in college chemistry students. Stress, 14(5), 557-566. http://dx.doi.org/10.3109/10253890.2011.571322

Benbenutty, H., Cleary, T.J. y Kitsantas, A. (2014). Applications of Self-Regulated Learning across Diverse Disciplines. Charlote, NC: Information Age Publishing, Inc.

Berry D.M. y York, K. (2010). Depression and religiosity and/or spirituality in college: a longitudinal survey of students in the USA. Nurs Health Sci, 13(1), 76-83. http://dx.doi.org/10.1111/j.1442-2018.2011.00584.x

Biggs, J.B., Kember, D., \& Leung, D.Y.P. (2001). The Revised Two Factor Study Process Questionnaire: R-SPQ-2F. British Journal of Educational Psychology, 71, 133-149. http://dx.doi.org/10.1348/000709901158433

Bodenlos, J.S., Noonan, M. y Wells, S.Y. (2013). Mindfulness and alcohol problems in college students: the mediating effects of stress. J Am Coll Health, 61(6), 371-378. http://dx.doi.org/10.1348/000709901158433

Buckingham, S. y Deakin, R. (2012). Learning dispositions and transferable competencies: pedagogy, modelling and learning analytics. 2nd International Conference on Learning Analytics\& Knowledge, 29 Apr - 02 May 2012. Vancouver, Canadá: British Columbia. http://oro.open.ac.uk/32823/1/sbs-rdc-lak12-oro.pdf

Burris, J.L, Brechting, E.H., Salsman, J., et al. (2009). Factors associated with the psychological well-being and distress of university students. J. Am Coll Health, 57(5), 536-543. http://dx.doi.org/10.3200/JACH.57.5.536-544

Caldwell, K., Harrison, M., Adams M., et al. (2010). Developing mindfulness in college students through movement-based courses: effects on self-regulatory self-efficacy, 
mood, stress, and sleep quality. $J$ Am Coll Health, 58(5), 433-442. http://dx.doi.org/10.1080/07448480903540481

Christenson, S.L., Reschly, A. L. y Wylie, C. (2014). Handbook of Research on Student Engagement. New York: Springer.

Chou PC, Chao YM, Yang HJ, et al. (2011). Relationships between stress, coping and depressive symptoms among overseas university preparatory Chinese students: a cross-sectional study. BMC Public Health 11, 352- 367. http://dx.doi.org/10.1186/1471-2458-11-352

Conley, C.S., Travers, L.V. y Bryant F.B. (2013). Promoting psychosocial adjustment and stress management in first-year college students: the benefits of engagement in a psychosocial wellness seminar. $J$ Am Coll Health, 61(2), 75-86. http://dx.doi.org/10.1080/07448481.2012.754757

Costarelli, V. y Patsai, A. (2012). Academic examination stress increases disordered eating symptomatology in female university students. Eat Weight Disord, 17(3), 164-169. http://dx.doi.org/10.1007/BF03325343

Davis, M., McKey, M. y Eshelman, E.R. (1985). Técnicas de autocontrol emocional. Barcelona: Martínez Roca.

Day, V., McGrath, P.J. y Wojtowicz, M. (2013). Internet-based guided self-help for university students with anxiety, depression and stress: a randomized controlled clinical trial. Behav Res Ther, 51(7), 344-351. http://dx.doi.org/10.1016/j.brat.2013.03.003

de la Fuente, J. (2004). Factores motivacionales en el aprendizaje escolar. En M.V. Trianes y J.A. Gallardo (Coord.). Psicología de la Educación y del Desarrollo en contextos escolares (pp. 461-478). Madrid: Pirámide.

de la Fuente, J. (2008). Action-Emotion Style as Characteristic of Achievement Motivation in University Students. In A. Valle, J.C. Núñez, R. González- Cabanach, J.A. GonzálezPienda y S. Rodríguez (eds.), Handbook of Instructional Resources and Their Applications in the Classroom (pp. 297-310). New York: Nova Science Publishers.

de la Fuente, J. (2014a). Competence model for learning, studing and achievement with stress: self-help model for universities, students and professional examinations. Almería (Spain): Education \& Psychology I+D+I, e-Publishing Series I+D+I.

de la Fuente, J. (2014b). Utilidad de e-Afrontamiento del Estrés Académico. Universidad de Almería. RPI en trámite.

de la Fuente, J. y Cardelle-Elawar, M.C. (2009). Research on action-emotion style and study habits: Effects of individual differences on learning and academic performance of 
undergraduate students. Learning \& Individual Differences, 19(5), 567-576. http://dx.doi.org/10.1016/j.lindif.2009.07.009

de la Fuente, J. y Cardelle-Elawar, M. (2011). Personal Self-Regulation and Coping Style in University Students. En L.B. Martin y R.A. Nichelson, Psychology of individual Differences (pp. 171-182). New York: Nova Science Publisher.

de la Fuente, J., Franco, C. y Mañas, I. (2010). Efectos de un programa de entrenamiento en conciencia plena (midfullness) en el estado emocional de estudiantes universitarios. Estudios sobre Educación, 19, 31-52.

de la Fuente, J., García-Berbén, A.B., \& Zapata, L. (2013). How regulatory teaching impacts university students' perceptions of the teaching-learning process: The role of teacher training. Infancia $\quad y \quad$ Aprendizaje, $36(3), \quad 375-385$. http://dx.doi.org/10.1174/021037013807533016

de la Fuente, J., Martínez-Vicente, J.M., Salmerón, J. L., Vera, M. M. \& Cardelle-Elawar, M. (in review). Action-emotion Style, Learning Approach, and Coping Strategies, in undergraduate University Students. Anales de Psicología.

de la Fuente, J., Sander, P., \& Putwain, D. (2013). Relationship between Undergraduate Student Confidence, Approach to Learning and Academic Performance: The role of gender. Journal of Psychodidactics, 18(2), 373-391.

de la Fuente, J., Solinas, G., Fadda, T. y Zapata, L. (in review). Improving mental health through an online self-asessment and self-help e-Utility in University students. En Maria M. Cruz-Cunha e Isabel Miranda (Eds.), Encyclopedia of e-Health and Telemedicine. NY: IGI Global.

de la Fuente, J., Zapata, L., Martínez-Vicente, J.M., Cardelle-Elawar, M., Sander, P.; Justicia, F., Pichardo, M.C., \& García-Berbén, A.B. (2012) Regulatory Teaching and SelfRegulated Learning in College Students: Confirmatory Validation Study of the IATLP Scales. Electronic Journal of Research in Educational Psychology 10(2), 839-866.

de la Fuente, J., Zapata, L., Martínez-Vicente, J.M., Sander, P. y Putwain, D. (2015). Personal Self-Regulation, Self-regulated Learning and Coping Strategies, in University Contexts with Stress. En A. Peña-Lara (ed.), Metacognition: Fundaments, Applications and Trends. A Profile of Currents-State-of-the-Art (chapter 9). New York: Springer.

Gonzalez, A., Paoloni, V., Donolo, D. et al. (2012). Motivational and emotional profiles in university undergraduates: a self-determination theory perspective. Span J Psychol, 15(3) 1069-1080. http://dx.doi.org/10.5209/rev_SJOP.2012.v15.n3.39397 
Gulewitsch MD, Enck P, Schwille-Kiuntke J, et al. (2013). Mental Strain and Chronic Stress among University Students with Symptoms of Irritable Bowel Syndrome. Gastroenterol Res Pract, 20, 65-74.

Hamaideh, S.H. (2011). Stressors and reactions to stressors among university students. Int J Soc Psychiatry, 57(1), 69-80. http://dx.doi.org/10.1177/0020764009348442

Hamdan-Mansour, A.M., Puskar, K. y Bandak, A.G. (2009). Effectiveness of cognitivebehavioral therapy on depressive symptomatology, stress and coping strategies among Jordanian university students. Issues Ment Health Nurs, 30(3), 188-196. http://dx.doi.org/10.1080/01612840802694577

Hartley, M.T. (2011). Examining the relationships between resilience, mental health, and academic persistence in undergraduate college students. J Am Coll Health, 59(7), 596604. http://dx.doi.org/10.1080/07448481.2010.515632

Lala, A., Bobirnac, G. y Tipa, R. (2010). Stress levels, alexithymia, type A and type C personality patterns in undergraduate students. J Med Life, 3(2), 200-205.

Largo-Wight, E., Peterson, P.M y Chen, W.W. (2005). Perceived problem solving, stress, and health among college students. Am J Health Behav, 29(4), 360-370. http://dx.doi.org/10.5993/AJHB.29.4.8

Law, D.W. (2007). Exhaustion in university students and the effect of coursework involvement. $\quad J \quad A m$ Coll Health, 55(4), 239-245. http://dx.doi.org/10.3200/JACH.55.4.239-245

Lorenz, V.R., Benatti, M.C. y Sabino, M.O. (2010). Burnout and stress among nurses in a university tertiary hospital. Rev Lat Am Enfermagem, 18(6), 1084-1091.

Malinauskas, R., Malinauskiene, V., Dumciene, A. (2010). Burnout and perceived stress among university coaches in Lithuania. $J$ Occup Health, 52(5), 302-307. http://dx.doi.org/10.1539/joh.O10006

Mateu, García-Renedo, Caballer y Gil-Beltrán (2010). Traducción y adaptación del CD-RISC (Connor y Davison, 2003). Universidad de Castellón.

McKay, M., Davis, M. y Fanning, P. (1985). Técnicas cognitivas para el tratamiento del estrés. Barcelona: Martínez-Roca.

O'Donovan, A. y Hughes, B. (2007). Social support and loneliness in college students: effects on pulse pressure reactivity to acute stress. Int J Adolesc Med Health, 19(4), 523-528. http://dx.doi.org/10.1515/IJAMH.2007.19.4.523 
Perera, B., Torabi, M. y Kay, N.S. (2011). Alcohol use, related problems and psychological health in college students. Int $J$ Adolesc Med Health, 23(1), 33-37. http://dx.doi.org/10.1515/IJAMH.2011.006

Pettit, M.L. y De Barr, K.A. (2011). Perceived stress, energy drink consumption, and academic performance among college students. J Am Coll Health, 59(5), 335-341. http://dx.doi.org/10.1080/07448481.2010.510163

Pichardo, M.C., Justicia, F., De la Fuente, J., Martínez-Vicente, J.M. y García-Berbén, A.B. (2014). Factor Structure of the Self-Regulation Questionnaire (SRQ) at Spanish Universities. The Spanish Journal of Psychology, 17, e62, 1-8.

Pintrich, P. (2004). A conceptual framework for assessing motivation and self-regulated learning in college students. Educational Psychology Review, 16, 385-407. http://dx.doi.org/10.1007/s10648-004-0006-x

Putwain, D., Sander, P. y Larkin, D. (2013). Academic self-efficacy in study-related skills and behaviours: relations with learning-related emotions and academic success. $\mathrm{Br} J$ Educ Psychol, 83(4), 633-650. http://dx.doi.org/10.1111/j.2044-8279.2012.02084.x

Regehr C, Glancy D, Pitts A. (2013). Interventions to reduce stress in university students: a review and meta-analysis. $J$ Affect Disord, 148(1), 1-11. http://dx.doi.org/10.1016/j.jad.2012.11.026

Romero, C., Master, A., Paunesku, D., et al. (2014). Academic and emotional functioning in middle school: The role of implicit theories. Emotion, 14(2), 227-234. http://dx.doi.org/10.1037/a0035490

Salmerón, J.L. (2014). Estilo de Acción-Emoción, estrategias de afrontamiento y resiliencia, en contextos estresantes universitarios de enseñanza-aprendizaje. Universidad de Almería: Tesis Doctoral no publicada.

Sander, P., De la Fuente, J., Stevenson, K. y Jones, T. (2011). A validation of the Academic Behavioural Confidence Scale with Spanish Psychology Students. Psychology Learning and Teaching, 10(1), 11-24. http://dx.doi.org/10.2304/plat.2011.10.1.11

Serlachius, A., Hamer, M. y Wardle, J. (2007). Stress and weight change in university students in the United Kingdom. Physiol Behav., 92(4), 548-53. http://dx.doi.org/10.1016/j.physbeh.2007.04.032

Sawatzky, R.G., Ratner, P.A., Richardson, C.G. et al. (2012). Stress and depression in students: the mediating role of stress management self-efficacy. Nurs Res, 61(1), 1321. http://dx.doi.org/10.1097/NNR.0b013e31823b1440 
Shamsuddin, K., Fadzil, F., Ismail, W, S., et al. (2013). Correlates of depression, anxiety and stress among Malaysian university students. Asian J Psychiatr, 6(4), 318-23. http://dx.doi.org/10.1016/j.ajp.2013.01.014

Small, T. \& Deakin Crick, R. (2008). The ELLI Projecy 'Learning and Self-Awareness: An Enquiry into Personal Development in Higher Education'. Bristol: Vital Partnership and Bristol University. Available at http://www.northumbria.ac.uk/sd/central/ar/lts/ltselli

Sulkowski, M.L., Dempsey, J. y Dempsey, A.G. (2011). Effects of stress and coping on binge eating in female college students. Eat Behav, 12(3), 188-191. http://dx.doi.org/10.1016/j.eatbeh.2011.04.006

Tavolacci, M.P., Ladner, J., Grigioni, S., et al. (2013). Prevalence and association of perceived stress, substance use and behavioral addictions: a cross-sectional study among university students in France, 2009-2011. BMC Public Health, 13,724-755. http://dx.doi.org/10.1186/1471-2458-13-724

Verger, P., Combes, J.B., Kovess-Masfety, V., et al. (2009). Psychological distress in first year university students: socioeconomic and academic stressors, mastery and social support in young men and women. Soc Psychiatry Psychiatr Epidemiol, 44(8), 643650. http://dx.doi.org/10.1007/s00127-008-0486-y

Villavicencio, F.T. y Bernardo, A.B. (2013). Positive academic emotions moderate the relationship between self-regulation and academic achievement. Br J Educ Psychol, 83(2), 329-340. http://dx.doi.org/10.1111/j.2044-8279.2012.02064.x

Weiner, B. (2005). Motivation from an attributional perspective and the social psychology of perceived competence. En A. Elliot \& C. S. Dweck (Eds.), Handbook of competence and motivation (pp. 73-84). New York, NY: Guilford Press.

Williamson, K.J., Coughlin, A., Small, T. y Thompson, J. (2011). My Learning Power. Using ELLI in Higher Education. A Guide to Understanding Learning Power withthe Effective Lifelong Learning Inventory - ELLI. A product of the Dispositions to Stay and Succeed Project.

https://www.heacademy.ac.uk/sites/default/files/Northumbria_ELLI_Dec_11_1.pdf

Zunhammer, M., Eberle, H. y Eichhammer, P. et al. (2013). Somatic symptoms evoked by exam stress in university students: the role of alexithymia, neuroticism, anxiety and depression. PLoS 8(12), pe84911. http://dx.doi.org/10.1371/journal.pone.0084911

Zimmerman, B.J. y Labuhn. A. S. (2012). Self-regulation of Learning: Process Approaches to Personal Development. In K.R. Harris, S. Graham, \& T. Urdam (Eds.), APA 
Jesús de la Fuente et al.

Educational Psychology Handbook (vol. 1, pp. 339-425). Washington: American Psychological Association. 